Article

\title{
On the Nature of $\gamma$-th Arithmetic Zeta Functions
}

\author{
Pavel Trojovský
}

Department of Mathematics, Faculty of Science, University of Hradec Králové, 50003 Hradec Králové, Czech Republic; pavel.trojovsky@uhk.cz; Tel.: +420-49-333-2860

Received: 10 April 2020; Accepted: 5 May 2020; Published: 8 May 2020

check for updates

\begin{abstract}
Symmetry and elementary symmetric functions are main components of the proof of the celebrated Hermite-Lindemann theorem (about the transcendence of $e^{\alpha}$, for algebraic values of $\alpha$ ) which settled the ancient Greek problem of squaring the circle. In this paper, we are interested in similar results, but for powers such as $e^{\gamma \log n}$. This kind of problem can be posed in the context of arithmetic functions. More precisely, we study the arithmetic nature of the so-called $\gamma$-th arithmetic zeta function $\zeta_{\gamma}(n):=n^{\gamma}\left(=e^{\gamma \log n}\right)$, for a positive integer $n$ and a complex number $\gamma$. Moreover, we raise a conjecture about the exceptional set of $\zeta_{\gamma}$, in the case in which $\gamma$ is transcendental, and we connect it to the famous Schanuel's conjecture.
\end{abstract}

Keywords: symmetry; zeta arithmetic function; transcendental numbers; Hermite-Lindemann theorem; Schanuel's conjecture

MSC: Primary 11J81; Secondary 11A25

\section{Introduction}

An arithmetic function is a complex-valued function whose domain is the set of natural numbers $\mathbb{N}$. The set $A$ of all arithmetic functions forms a ring with respect to addition $(+)$ and the Dirichlet convolution $(*)$, see [1,2] and some new results in [3-7]. Recall that the Dirichlet convolution of $f, g \in A$ is defined by

$$
(f * g)(n)=\sum_{d \mid n} f(d) g(n / d),
$$

where the sum extends over all positive divisors $d$ of $n$. A set of arithmetic functions $f_{1}, \ldots, f_{m}$ is said to be $*$-algebraically independent over $\mathbb{C}$ if there does not exist a non-zero polynomial $P$ with complex coefficients such that

$$
P\left(f_{1}, \ldots, f_{m}\right):=\sum_{(i)} a_{(i)} f_{1}^{* i_{1}} * \cdots * f_{m}^{* i_{m}}=0,
$$

where $a_{(i)} \in \mathbb{C}$ and $f^{* i}=f * \cdots * f(i$ times $)$.

Let $\gamma$ be a complex number. The $\gamma$-th arithmetic zeta function is defined as

$$
\zeta_{\gamma}(n)=n^{\gamma}
$$

Carlitz [8] showed that $\zeta_{0}, \ldots, \zeta_{r}$ are $*$-algebraically independent over $\mathbb{C}$, for any integer $r \geq 1$. A generalization for these Carlitz's result can be found in [9]. Our interest starts with the following definition:

Definition 1. Le $f: D \rightarrow \mathbb{C}$ be a function, with $D \cap \overline{\mathbb{Q}} \neq \varnothing$. We define the exceptional set of $f$ to be

$$
S_{f}=\{\alpha \in D \cap \overline{\mathbb{Q}}: f(\alpha) \in \overline{\mathbb{Q}}\} .
$$


Huang, Marques and Mereb [10] proved that for any $S \subseteq \overline{\mathbb{Q}}$, there exist uncountably many transcendental entire functions $f$ for which $S_{f}=S$.

In this work, we study the possible exceptional sets of $\gamma$-th arithmetic zeta functions.

\section{Algebraic Values of $\zeta_{\gamma}$}

We are interested in studying the set $S_{\zeta_{\gamma}}=\left\{\alpha \in \mathbb{N}: \zeta_{\gamma}(\alpha) \in \overline{\mathbb{Q}}\right\}$. For convenience, we write $S_{\gamma}$ instead of $S_{\zeta_{\gamma}}$. First, we point out to some simple facts (which hold for any $\gamma \in \mathbb{C}$ ):

- $S_{\gamma} \neq \varnothing$, since $1 \in S_{\gamma}$;

- If $n \in S_{\gamma}$, then $n^{k} \in S_{\gamma}$ for all $k \geq 1$;

- If $n \notin S_{\gamma}$, then $n^{k} \notin S_{\gamma}$ for all $k \geq 1$;

- If $n \in S_{\gamma}$ and $m \notin S_{\gamma}$, then $m n \notin S_{\gamma}$;

- If $Q \in \mathbb{Q} \backslash\{0\}$, then $S_{\gamma}=S_{Q \gamma}$.

Now, we shall split our study according to the arithmetic nature of $\gamma$.

\subsection{The Number $\gamma$ Is Algebraic}

For the rational case we use the following lemma

Lemma 1. Let $r$, s be any rational numbers. Then $r^{s}$ is an algebraic number.

Proof. Set $r=a / b$ and $s=m / n$. If $r^{s}=T \notin \overline{\mathbb{Q}}$, then we would have that $(a / b)^{m}=T^{n} \notin \overline{\mathbb{Q}}$. So $r^{s} \in \overline{\mathbb{Q}}$.

For the other case, we recall a well-known result

Theorem 1 (Gelfond-Schneider). If $\alpha \in \overline{\mathbb{Q}} \backslash\{0,1\}$ and $\beta \in \overline{\mathbb{Q}} \backslash \mathbb{Q}$, then $\alpha^{\beta} \notin \overline{\mathbb{Q}}$.

The proof of this theorem can be found in [11,12].

We state our first result on the exceptional sets of $\zeta_{\gamma}$ whose proof follows from Lemma 1 and by the Gelfond-Schneider theorem.

Proposition 1. Let $\gamma$ be an algebraic number.

(i) If $\gamma \in \mathbb{Q}$, then $S_{\gamma}=\mathbb{N}$;

(ii) If $\gamma \in \overline{\mathbb{Q}} \backslash \mathbb{Q}$, then $S_{\gamma}=\{1\}$.

\subsection{The Number $\gamma$ Is Transcendental}

For this case, we need to recall some concepts:

Definition 2. The complex numbers $x_{1}, \ldots, x_{m}$ are said to be multiplicatively independent, when the relation $x_{1}^{a_{1}} \cdots x_{m}^{a_{m}}=1$ with integral exponents $a_{1}, \ldots, a_{m}$ implies that $a_{1}=\cdots=a_{m}=0$. Otherwise, they are called multiplicatively dependent.

Example 1. The set of all prime numbers is multiplicatively independent (i.e., any finite subset of primes is multiplicatively independent). Furthermore, any set algebraically independent is multiplicatively independent.

Remark 1. The numbers $x_{1}, \ldots, x_{m}$ are multiplicatively independent if and only if $\log x_{1}, \ldots, \log x_{m}$ are $\mathbb{Q}$-linearly independent.

Now, recall the Lindemann theorem: if $t \in \overline{\mathbb{Q}} \backslash\{0\}$, then $e^{t}$ is transcendental. This important result implies, in particular, in the transcendence of $\pi$. Since any transcendental number cannot be 
constructed by using ruler and compass, the transcendence of $\pi$ (and so $\sqrt{\pi}$ ) settled the old Greek problem of the quadrature of the circle by showing that this task is impossible. For the case in which $t$ is transcendental, not much is known (e.g., is $e^{e}$ a transcendental number? So far, this problem remains open). However, a remarkable advance in this direction was provided in the next result.

Theorem 2 (Six Exponential Theorem). Let $\beta_{1}, \beta_{2}$ be complex numbers, linearly independent over $\mathbb{Q}$, and let $z_{1}, z_{2}, z_{3}$ be complex numbers, also linearly independent over $\mathbb{Q}$. Then at least one of the numbers

$$
e^{\beta_{1} z_{1}}, e^{\beta_{1} z_{2}}, e^{\beta_{1} z_{3}}, e^{\beta_{2} z_{1}}, e^{\beta_{2} z_{2}}, e^{\beta_{2} z_{3}}
$$

is transcendental.

This result was firstly explicitly stated and proved independently by Lang [13] and Ramachandra [14,15].

Now, we are able to state our next result:

Proposition 2. Let $\gamma$ be a transcendental number. Then any three different numbers in $S_{\gamma}$ must be multiplicatively dependent.

Proof. Let $n_{1}, n_{2}, n_{3} \in \mathbb{N}$ be multiplicatively independent numbers, so $\log n_{1}, \log n_{2}, \log n_{3}$ are linearly independent over $\mathbb{Q}$. Furthermore, $1, \gamma$ are linearly independent over $\mathbb{Q}$. Therefore, by Theorem 2 , there exists at least one transcendental number among

$$
e^{\log n_{1}}, e^{\log n_{2}}, e^{\log n_{3}}, e^{\gamma \log n_{1}}, e^{\gamma \log n_{2}}, e^{\gamma \log n_{3}} .
$$

Hence at least one of the numbers $n_{1}^{\gamma}, n_{2}^{\gamma}, n_{3}^{\gamma}$ is transcendental. In other words, at least one of the numbers $n_{1}, n_{2}, n_{3}$ is not in $S_{\gamma}$.

As consequences, we have:

Corollary 1. If $\gamma$ is a transcendental number, then $\mathbb{N} \backslash S_{\gamma}$ is an infinite set.

Proof. Let $p$ be a prime number, since $p, p^{2}$ and $p^{3}$ are multiplicatively dependent, then, by Proposition 2, at least one of the numbers $p, p^{2}, p^{3}$ must belong to $\mathbb{N} \backslash S_{\gamma}$. So, this set is infinite, because the existence of infinitely many primes.

Corollary 2. We have $S_{\gamma}=\mathbb{N}$ if and only if $\gamma \in \mathbb{Q}$.

Proof. The 'if' part is the item (i) of Proposition 1. The hypothesis in the 'only if' part, is that $S_{\gamma}=\mathbb{N}$. Then $\gamma$ cannot be transcendental (by Corollary 1). Furthermore, if $\gamma \in \overline{\mathbb{Q}} \backslash \mathbb{Q}$, we would have $S_{\gamma}=\{1\}$, by Proposition 1 (ii). In conclusion, $\gamma$ is a rational number.

Another consequence of Theorem 2 is the following result:

Proposition 3. If $\gamma$ is an irrational number and $A \subseteq \mathbb{N}$ is a set of multiplicatively independent numbers, then

$$
\#\left(S_{\gamma} \cap A\right) \leq 2 .
$$

Proof. Suppose, towards a contradiction, that $\#\left(S_{\gamma} \cap A\right) \geq 3$. If $a_{1}, a_{2}, a_{3} \in S_{\gamma} \cap A$, then $\log a_{1}, \log a_{2}$, $\log a_{3}$ are linearly independent over $\mathbb{Q}$ (since every finite subset of $A$ is multiplicatively independent). 
Furthermore, $1, \gamma$ are linearly independent over $\mathbb{Q}$ (since $\gamma$ is irrational). Therefore, by Theorem 2 , there exists at least one transcendental number among

$$
e^{\log a_{1}}, e^{\log a_{2}}, e^{\log a_{3}}, e^{\gamma \log a_{1}}, e^{\gamma \log a_{2}}, e^{\gamma \log a_{3}} .
$$

However, these numbers are

$$
a_{1}, a_{2}, a_{3}, a_{1}^{\gamma}, a_{2}^{\gamma}, a_{3}^{\gamma}
$$

which are all algebraic (since $a_{i} \in S_{\gamma}$ ). This contradicts Theorem 2 and then the proof is complete.

As an immediate consequence, we have:

Corollary 3. There are at most two distinct prime $p$ and $q$ such that both $p^{\pi}$ and $q^{\pi}$ are algebraic numbers.

Remark 2. We remark that it is really expected that any number of the form $n^{\pi}$, with $n>1$ integer, is transcendental. This is a consequence of Schanuel's conjecture (see Proposition 9 in the next section).

The conclusion of Proposition 3 is the best possible that we can prove in an unconditional way. However, there is a conjecture which allows us to prove the sharpest related result.

Conjecture 1 (Four exponential conjecture). Let $\beta_{1}, \beta_{2}$ be complex numbers, linearly independent over $\mathbb{Q}$, and let $z_{1}, z_{2}$ be complex numbers, also linearly independent over $\mathbb{Q}$. Then at least one of the numbers

$$
e^{\beta_{1} z_{1}}, e^{\beta_{1} z_{2}}, e^{\beta_{2} z_{1}}, e^{\beta_{2} z_{2}}
$$

is transcendental.

So, with the same ideas of the proof of Proposition 3, we can obtain:

Proposition 4. Suppose that Conjecture 1 holds. If $\gamma$ is an irrational number and $A \subseteq \mathbb{N}$ is a set of multiplicatively independent numbers, then

$$
\#\left(S_{\gamma} \cap A\right) \leq 1 .
$$

Remark 3. In fact, the previous result is sharp in the sense that we can not obtain that $\#\left(S_{\gamma} \cap A\right)=0$ in the general case. For example, if $\gamma=\log 3 / \log 2$ and $A=\{2\}$, then $\#\left(S_{\gamma} \cap A\right)=1$.

Now, note that when $\gamma \notin \overline{\mathbb{Q}}$, then we can have that $S_{\gamma}$ is an infinite set. For instance, $\left\{2^{k}: k \geq\right.$ $0\} \subseteq S_{\frac{\log 3}{\log 2}}$. This simple example encourage us to making a related conjecture, which, in particular, implies that $S_{\frac{\log 3}{\log 2}}=\left\{2^{k}: k \geq 0\right\}$.

Conjecture 2. If $\gamma \notin \mathbb{Q}$, then there exists $B \in \mathbb{N}$ such that $S_{\gamma}=\left\{B^{k}: k \geq 0\right\}$.

When $\gamma \in \overline{\mathbb{Q}} \backslash \mathbb{Q}$, then $B=1$. For the general case we have a hard problem, because until now we do not know the arithmetic nature of at least one of numbers of the form $n^{\pi}$ (surely for $n \neq 1$ ). So, it would be great to prove this conjecture, but unfortunately this problem seems to be out of reach for the current level of mathematics. Thus, instead of that, in next section we relate the well-known Schanuel's conjecture with the previous conjecture.

\section{Schanuel's Conjecture Versus Exceptional Set of $\zeta_{\gamma}$}

We recall the statement of Schanuel's conjecture which is one of the most important open problem in transcendental number theory. For ease of notation, we denote $\#\left\{x_{1}, \ldots, x_{m}\right\}$ as the 
transcendence degree (over $\mathbb{Q}$ ) of the field $\mathbb{Q}\left(x_{1}, \ldots, x_{m}\right)$ (in this case, the transcendence degree is the largest cardinality of an algebraically independent subset of $\left.\left\{x_{1}, \ldots, x_{m}\right\}\right)$.

Conjecture 3 (Schanuel). Let $x_{1}, \ldots, x_{n}$ be $\mathbb{Q}$-linearly independent complex numbers. Then

$$
\#\left\{x_{1}, \ldots, x_{n}, e^{x_{1}}, \ldots, e^{x_{n}}\right\} \geq n .
$$

Schanuel's conjecture implies many famous theorems and conjectures about transcendental numbers. For an interesting consequence, see [16]. From now on, we use this conjecture to prove some interesting facts on the exceptional set of the functions $\zeta_{\gamma}$.

Proposition 5. Suppose that Schanuel's conjecture holds. If $n \geq 2$ and $\gamma_{1}, \ldots, \gamma_{k}$ are algebraic numbers such that $1, \gamma_{1}, \ldots, \gamma_{k}$ are linearly independent over $\mathbb{Q}$, then the numbers $\log n, \zeta_{\gamma_{1}}(n), \ldots, \zeta_{\gamma_{k}}(n)$ are algebraically independent.

Proof. Since $\log n, \gamma_{1} \log n, \ldots, \gamma_{k} \log n$ are linearly independent over $\mathbb{Q}$, then, by Schanuel's conjecture,

$$
\#\left\{\log n, \gamma_{1} \log n, \ldots, \gamma_{k} \log n, n, \zeta_{\gamma_{1}}(n), \ldots, \zeta_{\gamma_{k}}(n)\right\} \geq k+1 .
$$

However $n, \gamma_{1}, \ldots, \gamma_{k} \in \overline{\mathbb{Q}}$ and then $\log n, \zeta_{\gamma_{1}}(n), \ldots, \zeta_{\gamma_{k}}(n)$ must be algebraically independent.

Proposition 6. Suppose that Schanuel's conjecture holds. If $\gamma \notin \overline{\mathbb{Q}}$ and $n_{1}, \ldots, n_{k} \in \mathbb{N}$ are multiplicatively independent, then

$$
\#\left\{\zeta_{\gamma}\left(n_{1}\right), \ldots, \zeta_{\gamma}\left(n_{k}\right)\right\} \geq k-1
$$

Proof. Since $n_{1}, \ldots, n_{k}$ are multiplicatively independent, then $\log n_{1}, \ldots, \log n_{k}$ are linearly independent over $\mathbb{Q}$. Next, let us order the numbers $\log n_{1}, \ldots, \log n_{k}$ in a such way that $\left\{\log n_{1}, \ldots, \log n_{k}, \gamma \log n_{1}, \ldots, \gamma \log n_{t}\right\}$ is a basis of the vector space generated by $\left\{\log n_{1}, \ldots, \log n_{k}, \gamma \log n_{1}, \ldots, \gamma \log n_{k}\right\}$ over $\mathbb{Q}$. Since $\gamma$ is a transcendental number, then there exists a transcendence basis $\left\{\log n_{i_{1}}, \ldots, \log n_{i_{s}}, \gamma\right\}$ of $\mathbb{Q}\left(\log n_{1}, \ldots, \log n_{k}, \gamma\right) \mid \mathbb{Q}$. Therefore $\log n_{1}, \ldots, \log n_{k}, \gamma \log n_{i_{1}}, \ldots, \gamma \log n_{i_{s}}$ are linearly independent over $\mathbb{Q}$ and then $s \leq t$. On the other hand, it follows from Schanuel's conjecture that

$$
\#\left\{\log n_{1}, \ldots, \log n_{k}, \gamma \log n_{1}, \ldots, \gamma \log n_{t}, n_{1}, \ldots, n_{k}, n_{1}^{\gamma}, \ldots, n_{t}^{\gamma}\right\} \geq t+k .
$$

So, $\#\left\{\log n_{1}, \ldots, \log n_{k}, \gamma \log n_{1}, \ldots, \gamma \log n_{k}, n_{1}^{\gamma}, \ldots, n_{k}^{\gamma}\right\} \geq t+k$. However

$$
\#\left\{\log n_{1}, \ldots, \log n_{k}, \gamma \log n_{1}, \ldots, \gamma \log n_{k}\right\}=\#\left\{\log n_{1}, \ldots, \log n_{k}, \gamma\right\}=s+1 .
$$

Hence, we obtain that $\#\left\{\zeta_{\gamma}\left(n_{1}\right), \ldots, \zeta_{\gamma}\left(n_{k}\right)\right\} \geq t+k-(s+1) \geq k-1$.

By considering that $\log n$ is a transcendental number for all $n \geq 2$ (by the Hermite-Lindemann theorem) and using a similar argument as in the previous proof, we get the next result:

Proposition 7. Suppose that Schanuel's conjecture holds. If $n \geq 2$ and $\gamma_{1}, \ldots, \gamma_{k}$ are linearly independent, then

$$
\#\left\{e^{\gamma_{1}}, \ldots, e^{\gamma_{k}}, \zeta_{\gamma_{1}}(n), \ldots, \zeta_{\gamma_{k}}(n)\right\} \geq k-1 .
$$

Now we prove that Conjecture 2 is also connected with the powerful Schanuel's conjecture.

Proposition 8. Schanuel's conjecture implies Conjecture 2. 
Proof. If $\gamma$ is an algebraic irrational number, then $S_{\gamma}=\{1\}$ and we take $B=1$. In the case in which $\gamma$ is transcendental, then we have two possibilities. The first one is $S_{\gamma}=\{1\}$ for which we choose $B=1$. Now, let us suppose that $S_{\gamma} \neq\{1\}$. In this case, we claim that there exists an integer $B \in S_{\gamma} \backslash\{1\}$ which is not a perfect power (i.e., $B \neq m^{t}$, for all integers $m \geq 1$ and $t>1$ ). Indeed, this follows because if $m^{t} \in S_{\gamma} \backslash\{1\}$, then $m$ also belongs to $S_{\gamma}$ (since $\left(m^{t}\right)^{\gamma}=\alpha \in \overline{\mathbb{Q}}$ implies $m^{\gamma}=\alpha^{1 / t} \in \overline{\mathbb{Q}}$ ). After taking this $B \in S_{\gamma}$ which is not a perfect power, it suffices to prove that $S_{\gamma}=\left\{B^{k}: k \geq 0\right\}$. Clearly, $\left\{B^{k}: k \geq 0\right\} \subseteq S_{\gamma}$. So, it remains to show that $S_{\gamma} \subseteq\left\{B^{k}: k \geq 0\right\}$. For that, take $n \in S_{\gamma}$. Note that the numbers $B, n$ are multiplicatively dependent (in fact, on the contrary, the Proposition 6 implies that at least on of the numbers $n^{\gamma}, B^{\gamma}$ is transcendental, which contradicts that fact that both $B$ and $n$ belong to $S_{\gamma}$ ). By the definition of multiplicative dependence, there are coprime integers $a, b$ such that $n=B^{a / b}$. Since $n$ is an integer and $\operatorname{gcd}(a, b)=1$, this forces $B$ to satisfy $B=m^{b}$, for some integer $m>1$. However, $B$ is not a perfect power and then $b=1$ yielding $n=B^{a} \in\left\{B^{k}: k \geq 0\right\}$. In conclusion $S_{\gamma} \subseteq\left\{B^{k}: k \geq 0\right\}$ which completes the proof.

Let $\gamma$ be a non-rational complex number. We call exceptional representative of $\gamma$ as the number $B=B(\gamma)$ in Conjecture 2, if any exists. Assuming that the Schanuel's conjecture holds, we just proved the existence of exceptional representative for any non-rational complex number. It is easy see that if the exceptional representative of $\alpha$ and $\beta$ are multiplicatively independent, then $S_{\alpha} \cap S_{\beta}=\{1\}$. We finish our work by expliciting $S_{\gamma}$, for some well-known $\gamma^{\prime}$ s.

Proposition 9. If Schanuel's conjecture holds, then the numbers $\zeta_{\pi}(n), \zeta_{e}(n), \zeta_{i}(n)$ are algebraically independent over $\mathbb{Q}(e, \pi)$, for all $n \geq 2$. In particular, $S_{\pi}=S_{i}=S_{e}=\{1\}$.

Proof. If $n \geq 2$, then $i \pi, \log n$ are $\mathbb{Q}$-linearly independent, so by Schanuel's conjecture $\#\{i \pi, \log n,-1, n\} \geq 2$. It follows that $\pi, \log n$ are algebraic independent. Thus $i \pi, \log n, \log \pi, \log \log n$

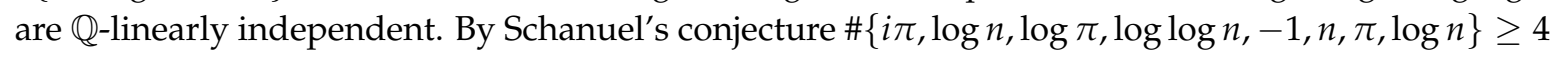
and then $1, i \pi, \log n, \log \pi, \log \log n$ are $\mathbb{Q}$-linearly independent. Again, by Schanuel's conjecture the numbers $i \pi, \log n, \log \pi, \log \log n$ are algebraically independent. Thus, for finishing, the numbers $i \pi, \pi \log n, e \log n, i \log n, \log \pi, \log \log n, 1, \log \pi, \log n$ are $\mathbb{Q}$-linearly independent. By Schanuel's conjecture the numbers $n^{\pi}, n^{e}, n^{i}$ are algebraically independent over $\mathbb{Q}(e, \pi)$.

\section{Conclusions}

In this paper we have been interested in the arithmetic nature of the arithmetic zeta functions $\zeta_{\gamma}(n)=n^{\gamma}$, where $n$ is a positive integer and $\gamma$ is a complex number. In particular, we study this nature for the case in which $\gamma$ is algebraic or transcendental. The main ingredients in the proofs are classical results on transcendence (such as, Lindemann, Gelfond-Schneider and the six exponential theorem) together with symmetry movements related to decrease (in size) a finite set in order to be able to apply some of our tools. We also pose a general conjecture and we show that it is a consequence of the important Schanuel's conjecture.

Funding: The author was supported by Project of Excelence PrF UHK No. 2213/2020, University of Hradec Králové, Czech Republic.

Acknowledgments: I would like to appreciate the help provided by Diego Marques from University of Brasilia in finalizing the text of this paper.

Conflicts of Interest: The author declares no conflict of interest.

\section{References}

1. Cashewll, E.D.; Everett, C.J. The ring of number-theoretic functions. Pac. J. Math. 1959, 9, 975-985. [CrossRef]

2. McCarthy, P.J. Introduction to Arithmetic Functions, 1st ed.; Springer: New York, NY, USA, 1985.

3. Nichita, F.F. On Transcendental Numbers. Axioms 2014, 3, 64-69. [CrossRef] 
4. Marcus, S.; Nichita, F.F. On Transcendental Numbers: New Results and a Little History. Axioms 2018, 7, 15. [CrossRef]

5. Hwang, K.-W.; Ryoo, C.S. Some Symmetric Identities for the Multiple $(p, q)$-Hurwitz-Euler eta Function. Symmetry 2019, 11, 645. [CrossRef]

6. Rong, J.; Xu, J. Three Results on the Nonlinear Differential Equations and Differential-Difference Equations. Mathematics 2019, 7, 539. [CrossRef]

7. Trojovský, P. Algebraic Numbers as Product of Powers of Transcendental Numbers. Symmetry 2019, 11, 887. [CrossRef]

8. Carlitz, L. Independence of arithmetic functions. Duke Math. J. 1952, 19, 65-70. [CrossRef]

9. Laohakosol, V.; Kongsakorn, K.; Leerawat, U. Algebraic independence test of arithmetic functions using Jacobians. J. Sci. Soc. Thail. 1989, 15, 133-138. [CrossRef]

10. Huang, J.; Marques, D.; Mereb, M. Algebraic values of transcendental functions at algebraic points. Bull. Aust. Math. Soc. 2010, 82, 322-327. [CrossRef]

11. Gelfond, A.O. Sur le septième problème de Hilbert. Investia Akad. Nauk. 1934, 7, 623-630.

12. Schneider, T. Transzendenzuntersuchungen periodischer funktionen: I transzendenz von potenzen; II transzendenzeigenschaften elliptischer funktionen. J. Reine Angew. Math. 1934, 172, 65-74.

13. Lang, S. Introduction to Transcendental Numbers, 1st ed.; Addison-Wesley Publishing Co.: Reading, MA, USA, 1966.

14. Ramachandra, K. Contributions to the theory of transcendental numbers I. Acta Arith. 1967, 14, 65-72. [CrossRef]

15. Ramachandra, K. Contributions to the theory of transcendental numbers II. Acta Arith. 1968, 14, 73-88. [CrossRef]

16. Cheng, C.; Dietel, B.; Herblot, M.; Huang, J.; Krieger, H.; Marques, D.; Mason, J.; Mereb, M.; Wilson, S.R. Some consequences of Schanuel's conjecture. J. Number Theory 2009, 129, 1464-1467. [CrossRef]

(C) 2020 by the author. Licensee MDPI, Basel, Switzerland. This article is an open access article distributed under the terms and conditions of the Creative Commons Attribution (CC BY) license (http://creativecommons.org/licenses/by/4.0/). 